\title{
CONTRIBUTION OF PILI OF S. PNEUMONIAE IN THE ONSET OF MENINGITIS
}

\author{
Jiménez-Munguía, I. ${ }^{1}$, Pulzová, L. ${ }^{1}$, Bhide, K. ${ }^{1}$, Čomor, L. ${ }^{1}$, Káňová, E. ${ }^{1}$ \\ Tomečková, Z. ${ }^{1}$; Širochmanová, I. ${ }^{1}$, Bhide, M. ${ }^{1,2}$ \\ ${ }^{1}$ Laboratory of Biomedical Microbiology and Immunology \\ University of Veterinary Medicine and Pharmacy, Kosice \\ ${ }^{2}$ Institute of Neuroimmunology of the Slovak Academy of Science, Bratislava, \\ Slovakia \\ sire.jm@hotmail.com
}

\begin{abstract}
Bacterial meningitis is a devastating worldwide disease. Half of the survivors of meningitis remain with permanent neurological sequelae. The pathogenesis of meningitis is based on a complex host-pathogen interaction. Streptococcus pneumoniae is a life-threatening neuroinvasive pathogen that asymptomatically colonizes the upper respiratory tract. Adherence of pneumococci to the host epithelium is a prerequisite in the onset of streptococcal infections; such adherence is favored by the formation of bacterial pili. In this article, we will describe the pneumococcal pili and its contribution to the onset of meningitis.
\end{abstract}

Key words: meningitis; pili; S. pneumoniae

\section{INTRODUCTION}

Streptococcus pneumoniae is a Gram-positive bacteria and one of the most common etiological agents of bacte- rial meningitis which is associated with high mortality and morbidity [30]. Pneumococci asymptomatically colonize the human nasopharynx in up to $40 \%$ of adults [19]. The infectivity of this pathogen and the development of protective immunity is poorly described in the current literature [25]. Meningitis-causing S. pneumoniae possess a set of antigenic structures, such as the cell wall components (i.e. teichoic acids, surface-associated proteins, adhesins and pili) that contribute to the bacterial invasion of the host epithelial lining [15].

In order to secure attachment on to host tissues, S. pneumoniae use adhesins normally located on its surface or at the end of long hair-like structures called pili [26]. Some strains of this pathogenic bacteria form pili, which play a role in biofilm formation, a characteristic life-style of the bacteria constituting the oral flora and are involved in primary colonization [5]. Pili are encoded within pathogenicity islands and differ among Gram-positive and Gramnegative bacteria. In the case of Gram-positive bacteria, protein subunits are covalently linked, chaperons are not required for assembly and tlp protein is not needed to initiate the formation of the pilus structure [26]. 


\section{Pili of Gram-positive bacteria}

Pili are long proteinaceous structures implicated in many functions such as adhesion to host cells, biofilm formation, DNA uptake and immune evasion. These structures were identified for the first time in the Gram-positive bacteria Corynebacterium diphtheria, and later in many others, such as S.pneumoniae [5]. The pili of Gram-positive bacteria are made of covalently linked pilins forming a string of beads of approximately $3 \mathrm{~nm}$ in diameter and various lengths ranging from 0.1 to $5 \mu \mathrm{m}$, although individual single pili of $9.5 \mathrm{~nm}$ diameter have been observed from cryo-EM on S. pneumoniae TIGR4 preparations [10].

The cell wall of Gram-positive bacteria is a surface structure, which promotes interactions with its environment and provides physical cell integrity [17]. The forma- tion of pili initiates at the plasma membrane in which the transpeptidase enzymes are located, and continues to the cell wall, where proteins are anchored to form hair-like structures. Via transpeptidation, sortases A, B, D and E anchor surface proteins to the cell wall by recognizing the conserved C-terminal LPxTG motif, meanwhile sortase C links proteins for the pili assembly (Figure 1)[5]. Cell wall decorators (i.e. all the chemical structures attached to the cell wall such as proteins) provide bacterial envelopes with species- and strain-specific properties. All of these structures, including the bacterial pili, contribute to bacterial virulence, or favor interactions with the host immune systems [17]. Specifically, the pili of S. pneumoniae becomes a key element that perform multiple functions during bacterial life cycles, such as host cell invasion, biofilm forma-

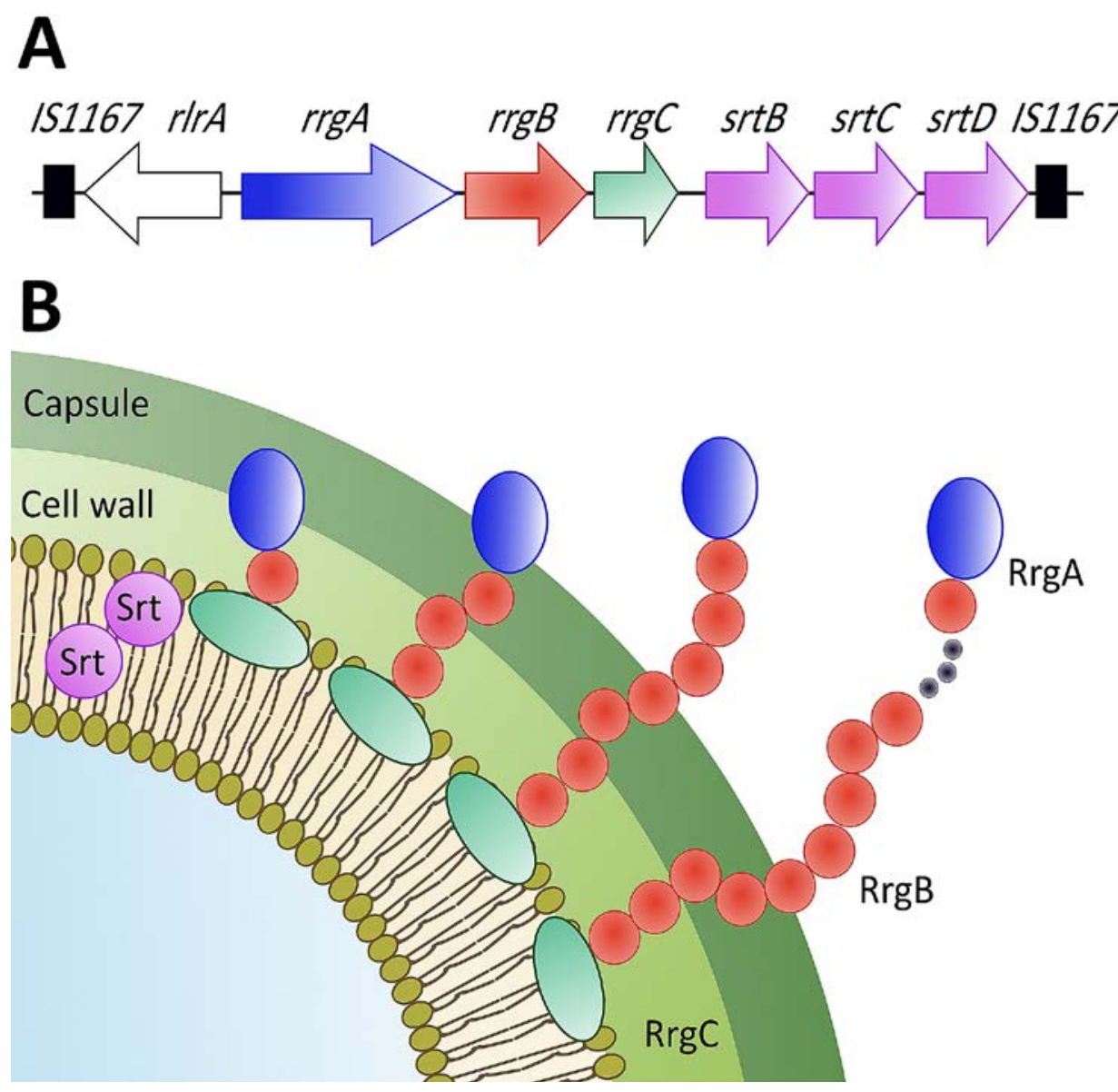

Fig. 1. Pili of S. pneumoniae

A) Organization of gene clusters encoding pili in the pathogenic strain S. pneumoniae TIGR4. Encoding genes for the following proteins are represented as arrows: sortases (violet), major pilin protein $\mathrm{RrgB}$ (orange), minor pilin proteins RrgA (blue) and RrgC (green). White arrow represents gene flanking the cluster; B) Biogenesis of pili of S. pneumoniae TIGR4. Sortases (green circles), located in the plasma membrane are responsible for anchoring pili to the cell wall. Anchor adhesin RrgC is shown as green oval, major pilin subunit RrgB is coloured in orange and minor adhesion RrgA is located in the tip of pili (blue oval). Figure adapted from Hilleringmann et al., 2009; Scott, Zähner, 2006 [26, 11] 
tion, cell aggregation, DNA transfer and twitching motility [11].

\section{Assembly of pilus in Streptococcus pneumoniae}

S. pneumoniae produces pili protruding from the bacterial cell surface. This structure is not always visible in all the cells from the same population [3]. Pili are composed of covalently linked pilin subunits. Each subunit has a LPxTG motif required for the covalent attachment to other pilin subunits and it is thought to ultimately be linked to the cell wall peptidoglycan by the housekeeping sortase A [1]. The sortase recognition motif LPxTG is included in the cell wall sorting signal (CWSS) region, together with a transmembrane anchor and a positively charged cytoplasmic tail [8]. The sortase-mediated pilus assembly was first demonstrated in C.diphtheria. The pilus assembly is performed on the bacterial surface once pilins are secreted across the plasma membrane via the Sec-dependent pathway. The nascent pilins are anchored in the membrane by its hydrophobic domain of the CWSS [5]. Transpeptidase enzymes responsible to link pilin subunits in S.pneumoniae are the sortases SrtC-1, SrtC-2, and SrtC-3 [8]. The pilus backbone is formed in a head-to-tail manner by the polymerization of the backbone pilin subunits, in which a lysine of the pilin motif of one subunit forms a covalent isopeptide bond with a threonine residue in the LPxTG of the next subunit.

The last step is carried out by the sortase A, in which the mature filament is covalently linked to the pepdidoglycan precursor lipid II, leading to the covalent attachment of the pilus to the cell wall. Additionally, one or two accessory pilins are integrated into individual pili. Minor pilin 1 containing the LPxTG motif are first integrated into the pilus, serving as pilus polymerization initiators and they are preferentially located at the pilus tip; meanwhile the Minor pilin 2 is often found at the base of the pilus and serves as an anchor [5].

\section{Structure of pili of Streptococcus pneumoniae}

S. pneumoniae expresses two types of pili. Pilus-1, encoded by pilus islet 1 , is composed of a backbone subunit $\mathrm{RrgB}$ and two ancillary proteins, RrgA and RrgC (Figure 1). Pilus-2 is encoded by the pilus islet 2 and is composed of a pitB backbone subunit $[1,11]$. The genes required for pilus production are located in a $12 \mathrm{~Kb}$ pathogenicity island (the rlrA islet) [27]. The pilus-1 expressed by S. pneumoni$a e$ is composed of one major ( $\operatorname{RrgB})$ and two minor ( $\operatorname{Rrg} \mathrm{A}$ and $\mathrm{RrgC}$ ) structural proteins. Filaments are formed by a single string of RrgB monomers, which are elongated with $\mathrm{RrgA}$ and $\mathrm{RrgC}$ according to their putative roles as adhesion and anchor to the cell wall surface, respectively (Figure 1) [10]. Moreover, a genomic region of S. pneumoniae codes for a second functional pilus. This region is composed of five genes, two of them coding for putative LPxTG-type surface-anchored proteins (pitB and pitA), the latter containing a stop codon, one gene encoding a signal peptidaserelated product ( $\operatorname{sipA}$ ), and two genes coding products displaying similarity to sortases (srtG1 and srtG2) [1]. PitB is suggested to be the most probable backbone of the fimbria containing stabilizing intramolecular isopeptide bonds and PitA, a putative ancillary protein [31].

\section{Biological functions of bacterial pili}

Bacterial pili are considered as surface-exposed virulence factors [14]. These structures appear crucial for the initiation of bacterial colonization inside the host and are involved in adhesion, recognition of host cell receptors, biofilm formation and evasion from the innate immune system, all of which contribute to infection [5]. The role of pili in adherence has been demonstrated in several Grampositive bacteria such as $C$. renale and $S$. agalactiae, which adhere to kidney or to human lung epithelial cells $[7,12]$. S. agalactiae expresses a pili composed of three structural subunits (PilA, PilB and PilC), of which PilB is the major backbone protein; PilA is an adhesive protein located at the tip of the pilus (AP-1), and PilC (AP-2) acts as pilus anchors [7].

In regard to $S$. pneumoniae, $\operatorname{RrgB}$ is the shaft protein and two accessory proteins, RrgA and RrgC, act as an adhesin and anchor, respectively [11]. Both PilA and RrgA adhesins exhibit a von Willebrand adhesion domain (VWA), which is important for their cell binding properties [14]. VWA-containing proteins are widely distributed among archae, bacteria and eukaryotes [23]. This domain found in eukaryotes is involved in interactions with the extracellular matrix (ECM) [28]. Likewise, some bacterial surface proteins specifically interact with extracellular matrix components such as fibronectin, fibrinogen, collagen, and heparin-related polysaccharides [22]. Similarly, RrgA has been shown to bind in a dose-dependent manner to the ECM compounds (e.g. fibrinogen, fibronectin, laminin and collagen I) and to recognize different receptors on their target cells $[10,14]$. Collagen-binding Microbial 
Surface Components Recognizing Adhesive Matrix Molecule (MSCRAMMs) represents a major class of adhesins contributing to host colonization. Pilus-associated adhesins often belong to the MSCRAMMs family, suggesting the importance of pili in bacterial adhesion to the extracellular matrix [5]. It has been demonstrated that there is markedly less adherence to human respiratory epithelial cells when the gene encoding for the RrgA pilin subunit of S. pneumoniae TIGR4 has been deleted [21].

Concerning the biofilm formation, the 3D-biofilm structure begins with a primary attachment of the bacterial surface. Then, interactions among bacteria are strengthened by long pilus structures that contribute to an irreversible attachment. Regularly, during infections $S$. pneumoniae exists in a sessile biofilm rather than in a planktonic form, except during sepsis or meningitis $[13,22]$. Specifically, it has been established the contribution of RrgA in biofilm formation once insertions into $\operatorname{rrgA}$ gene, encoding the PI-1 pilus adhesion, abrogated biofilm formation [20].

On the other hand, pili have also been related to the host immune responses modulation. Piliated strains of S. pneumoniae triggered higher pro-inflammatory TNF-a and IL-6 responses compared to the non-piliated counterpart during systemic infections. Finally, another pili-related function is the contribution to colonization. Pneumococci expressing PI-1 displayed advantages on colonization of the respiratory tract in mice after intranasal challenge in comparison to non-piliated TIGR4 mutant which had shown less virulence in the murine models of colonization, pneumonia and bacteraemia. Particularly, S. pneumoniae TIGR4 pilus-associated RrgA is critical for the colonization of the upper respiratory tract in mice [21].

\section{Colonization and neuroinvasion of $S$. pneumoniae}

S.pneumoniae colonizes the nasopharynx degrading the mucus viscosity by ex-olygosidases such as neuraminidase A (NanA), $\beta$-galactosidase (BgaA), N-acetylglucosaminidase (StrH), and neuraminidase B (NanB), which allows the pneumococci to persist in the airways [20]. It is believed that pneumococcal meningitis is acquired via this colonization, followed by bacteraemia and invasion of the Central Nervous System (CNS) [4]. Recent studies have reported that a high degree of bacteraemia is necessary but at times may not be sufficient enough for developing meningitis. Moreover, microbial binding to microvascular endothelial cells (BMEC), and invasion in this cell type is a prerequisite for the penetration of the blood-brain barrier (BBB). Alternatively, other routes of bacterial entry into the CNS have been associated with pneumococci. Such routes include spreading from contiguous sources of infection, or to enter into the CNS through a non-hematogenous route after intranasal inoculation in experimental animals or otitis media [9].

Pathogens causing meningitis share a common strategy to move from the mucosa into the brain through the blood stream. Many bacteria bind to extracellular matrix proteins (e.g. laminin, collagen or fibronectin), which facilitate the initial attachment preceding the actual invasion [16]. S. pneumoniae is thought to enter the CNS by crossing the $\mathrm{BBB}$ or the blood-cerebrospinal fluid (CSF) barrier by local tissue damage or by transcytosis through BMEC [6]. In order to enter the CNS, pneumococci possess an armory of virulence factors including surface proteins, polysaccharide capsule and cell wall components for invasion [16]. The capsule is a major virulence determinant due to its anti-phagocytic activity. Some surface-exposed proteins such as pneumococcal surface protein A (PspA) and $\mathrm{C}$ (PspC) are the best characterized as choline-binding proteins. PspA interferes with complement activation while PspC interacts with human immunoglobin A and with the polymeric immunoglobulin receptors, showing also antiphagocytic properties due to its capability to bind to complement C3 [18].

Pneumococci have the ability to bind to certain receptors located on the plasma membrane of epithelial and endothelial cells. This receptor-mediated binding facilitates bacterial translocation and the bacterial invasion through the human cell layers mice [21]. The binding of bacterial adhesins to specific host cell receptors may lead to a signal transduction resulting in tight bacterial attachment or internalization via host cells invasion [16]. Pneumococci could take advantage of an increased expression of cell adhesion molecules, generated during inflammation as a response of cell migration, attach to the platelet-activating factor (PAF) receptor and cross the $\mathrm{BBB}$ by transcellular mechanisms [24]. Once pneumococci enter the CNS, the brain's resident macrophages act as key effectors of initial innate immunity clearing the bacteria and recruiting peripheral blood cells to the site of infections [18]. Replication of pneumococci inside the subarachnoid space, simultaneously occurs with the release of bacterial products such as peptidoglycans, which are highly immunogenic and in- 
crease the inflammatory response. The initial pneumococcus sensing in the CNS is performed by Toll-like 2, 4 and 9 receptors which recognize peptidoglycan, pneumolysin, and CpG in the bacterial DNA, respectively [29].

\section{Role of S. pneumoniae pili in meningitis}

Pneumococcal meningitis is characterized by a high mortality rate $(20-30 \%)$ due to complications derived from an excessive immune response as well as damage by the pathogen itself. These complications include brain ede$\mathrm{ma}$, cerebral ischemia and increased intracranial pressure [16]. Expression of the pili is related with the pathogenicity of the pneumococci. Thirty percent of the S. pneumoniae strains, isolated from clinical environments are piliated and contain the rlrA genetic element [14].

The contribution of $S$. pneumoniae pili in meningitis has not been completely described. Pathogens-causing meningitis possess small size and this feature may facilitate their migration through the BBB. Pneumococci grow forming chains and sometimes they are found as individual cocci. Chain formation is thought to favor adherence to the epithelium [2]. In 2016, I ovin o et al. described the advantages that pili-expressing $S$. pneumoniae possess for the brain entry; mice infected with piliated S. pneumoniae TIGR4 had approximately $80 \%$ more pneumococci in the brain than mice infected with a non-piliated strain. The ability to enter the brain was also evaluated using TIGR4 $\Delta \operatorname{rrgA}$ and TIGR4 $\triangle$ rrgBC mutants, suggesting that RrgA allows bacterial binding to the $\mathrm{BBB}$ endothelium, and thereby promotes the entry of pneumococci into the brain. Despite whether or not S. pneumoniae forms either chains or biofilms in the majority of infections during meningitis, individual cells have been observed inside the brain more frequent than in the bloodstream. This fact suggests that even if the fraction of single cocci in circulation is low, they continuously seed the brain endothelium during infections [21].

\section{CONCLUSIONS}

In conclusion, bacterial pili play different roles during infections. Meningitis caused by pathogenic bacteria such as $S$. pneumoniae have been widely studied but the detailed mechanisms about how the bacteria enter into the brain still remain unknown. Pili in Gram-positive bacteria are well characterized but their interplay with the CNS, spe- cifically with cells from the neurovascular unit, needs to be investigated in order to decipher its contribution in the development of meningitis.

\section{ACKNOWLEDGEMENTS}

The study was supported by APVV-14-0218 and INFEKTZOON (Center of excellence for infections in animals and zoonoses, ITMS code: 26220120002, co-financed from the European structural funds) for support of this study.

\section{REFERENCES}

1. Bagnoli, F., Moschioni, M., Donati, C., Dimitrovska, V., Ferlenghi, I., Facciotti, C., et al., 2008: A second pilus type in Streptococcus pneumoniae is prevalent in emerging serotypes and mediates adhesion to host cells. J. Bacteriol., 190, 5480-5492.

2. Barichello, T., Generoso, J.S., Collodel, A., Moreira, A.P., Almeida, S.M. De., 2012: Pathophysiology of acute meningitis caused by Streptococcus pneumoniae and adjunctive therapy approaches. Arquivos de neuro-psiquiatria, 70, 366-372. http://www.ncbi.nlm.nih.gov/pubmed/22618789.

3. Barocchi, M. A., Ries, J., Zogaj, X., Hemsley, C., Albiger, B., Kanth, A., et al., 2006: A pneumococcal pilus influences virulence and host inflammatory responses. In Proc. Natl. Acad. Sci. USA. 103, 2857-2862. http://www.pubmedcentral.nih. gov/articlerender.fcgi?artid=1368962\&tool=pmcentrez\&rend ertype $=$ abstract $(4$ March 2015) .

4. Burnaugh, A.M., Frantz, L. J. and King, S. J., 2008: Growth of Streptococcus pneumoniae on human glycoconjugates is dependent upon the sequential activity of bacterial exoglycosidases. J. Bacteriol., 190, 221-230.

5. Danne, C., Dramsi, S., 2012: Pili of Gram-positive bacteria: Roles in host colonization. Research in Microbiology, 163, 645-658. http://dx.doi.org/10.1016/j.resmic.2012.10.012.

6. Doran, K.S., Fulde, M., Gratz, N., Kim, B.J., Nau, R., Prasadarao, N., et al., 2016: Host-pathogen interactions in bacterial meningitis. Acta Neuropathologica, 131, 185-209.

7. Dramsi, S., Caliot, E., Bonne, I., Guadagnini, S., Prévost, M.C., Kojadinovic, M., et al., 2006: Assembly and role of pili in group B streptococci. Molecular Microbiology, 60, 1401-1413.

8. El Mortaji, L., Fenel, D., Vernet, T., Di Guilmi, A.M., 2012: Association of RrgA and $\mathrm{RrgC}$ into the Streptococcus 
pneumoniae pilus by sortases C-2 and C-3. Biochemistry, 51, $342-352$.

9. Gurung, M., Moon, D. C., Choi, C. W., Lee, J.H., Bae, Y.C., Kim, J., et al., 2011: Staphylococcus aureus produces membrane-derived vesicles that induce host cell death. PLoS ONE, $6,1-8$.

10. Hilleringmann, M., Giusti, F., Baudner, B. C., Masignani, V., Covacci, A., Rappuoli, R., et al., 2008: Pneumococcal pili are composed of protofilaments exposing adhesive clusters of Rrg A. PLoS Pathogens, 4, e1000026.

11. Hilleringmann, M., Ringler, P., Müller, S. A., De Angelis, G., Rappuoli, R., Ferlenghi, I., et al., 2009: Molecular architecture of Streptococcus pneumoniae TIGR4 pili. The EMBO Journal, 28, 3921-3930. http://emboj.embopress.org/cgi/doi/ 10.1038/emboj.2009.360.

12. Honda, E., Yanagawa, R., 1978: Pili-mediated attachment of Corynebacterium renale to mucous membrane of urinary bladder of mice. Am. J. Vet. Res., 39, 155-158.

13. Iovino, F., Hammarlöf, D. L., Garriss, G., Brovall, S., Nannapaneni, P., Henriques-Normark, B., 2016: Pneumococcal meningitis is promoted by single cocci expressing pilus adhesin RrgA. J. Clin. Invest., 126, 2821-2826.

14. Izoré, T., Contreras-Martel, C., El Mortaji, L., Manzano, C., Terrasse, R., Vernet, T., et al., 2010: Structural basis of host cell recognition by the pilus adhesin from Streptococcus pneumoniae. Structure, 18, 106-115.

15. Jayaraman, R., 2011: Phase variation and adaptation in bacteria: A 'Red Queen's Race. Current Science, 100, 1163-1171.

16. Kim, K.S., 2008: Mechanisms of microbial traversal of the blood-brain barrier. Nature reviews. Microbiology, 6, 625-34. http://dx.doi.org/10.1038/nrmicro1952 (23 March 2016).

17. Marraffini, L.A., DeDent, A.C., Schneewind, O., 2006: Sortases and the art of anchoring proteins to the envelopes of Gram-positive bacteria. Microbiol. Mol. Biol. Rev., 70, 192221. http://mmbr.asm.org/cgi/doi/10.1128/MMBR.70.1.192221.2006.

18. Meli, D.N., Christen, S., Leib, S.L., Täuber, M.G., 2002: Current concepts in the pathogenesis of meningitis caused by Streptococcus pneumoniae. Cur. Opin. Infect. Dis., 15, 253-257. http://www.ncbi.nlm.nih.gov/pubmed/12015459 (15 January 2016).

19. Mook-Kanamori, B. B., Geldhoff, M., Der, T. Van, van der Poll, T. B., van de Eek, D., 2011: Pathogenesis and pathophysiology of pneumococcal meningitis. Clin. Microbiol. Rev., 24, 557-591.

20. Muñoz-Elías, E. J., Marcano, J., Camilli, A., 2008: Isolation of Streptococcus pneumoniae biofilm mutants and their char- acterization during nasopharyngeal colonization. Infect. Immun., 76, 5049-5061.

21. Nelson, A. L., Ries, J., Bagnoli, F., Dahlberg, S., Fälker, S., Rounioja, S., et al., 2007: $\operatorname{RrgA}$ is a pilus-associated adhesin in Streptococcus pneumoniae. Mol. Microbiol., 66, 329-340.

22. Oggioni, M.R., Trappetti, C., Kadioglu, A., Cassone, M., Iannelli, F., Ricci, S., et al., 2006: Switch from planktonic to sessile life: A major event in pneumococcal pathogenesis. Mol. Microbiol., 61, 1196-1210.

23. Ponting, C.P., Aravind, L., Schultz, J., Bork, P., Koonin, E. V., 1999: Eukaryotic signalling domain homologues in archaea and bacteria. Ancient ancestry and horizontal gene transfer. J. Mol. Biol., 289, 729-745. http://linkinghub.elsevier.com/retrieve/pii/S0022283699928279.

24. Ricci, S., Gerlini, A., Pammolli, A., Chiavolini, D., Braione, V., Tripodi, S. A., et al., 2013: Contribution of different pneumococcal virulence factors to experimental meningitis in mice. BMC Infect. Dis., 13, 444. http://www.pubmedcentral. nih.gov/articlerender.fcgi? artid=3848944\&tool=pmcentrez\& rendertype $=$ abstract.

25. Scheld, W. M., Whitley, R. J., Marra, C. M., 2004: Infections of the Central Nervous System. 1st edn., Williams \& Wilkins, China, $353 \mathrm{pp}$.

26. Scott, J.R., Zähner, D., 2006: Pili with strong attachments: Gram-positive bacteria do it differently. Mol. Microbiol., 62, $320-330$.

27. Spraggon, G., Koesema, E., Scarselli, M., Malito, E., Biagini, M., Norais, N., et al., 2010: Supramolecular organization of the repetitive backbone unit of the Streptococcus pneumoniae pilus. PLoS ONE, 5, e10919.

28. Springer, T.A., 2006: Complement and the multifaceted functions of VWA and integrin I domains. Structure, 14, $1611-161$.

29. Tuomanen, E. I., 1996: Molecular and cellular mechanisms of pneumococcal meningitis. Ann. NY Acad. Sci., 797, 42-52.

30. Van de Beek, D., Brouwer, M., Hasbun, R., Koedel, U., Whitney, C.G., Wijdicks, E., 2016: Community-acquired bacterial meningitis. Nat. Rev. Dis. Primers, 2, 1-20. http:// www.nature.com/articles/nrdp201674.

31. Zähner, D., Gandhi, A. R., Stuchlik, O., Reed, M., Pohl, J., Stephens, D. S., 2011: Pilus backbone protein PitB of Streptococcus pneumoniae contains stabilizing intramolecular isopeptide bonds. Biochem. Biophys. Res. Comm., 409, 526-531.

Received December 4, 2017

Accepted February 8, 2018 